\title{
DRIVER DROWSINESS IMMEDIATELY BEFORE CRASHES - A COMPARATIVE INVESTIGATION OF EEG PATTERN RECOGNITION
}

\author{
M. Golz ${ }^{1,3}$, D. Sommer ${ }^{1}$, U. Trutschel ${ }^{2,3}$, J. Krajewski ${ }^{4}$ \& B. Sirois ${ }^{2}$ \\ ${ }^{1}$ Faculty of Computer Science, University of Applied Sciences, Schmalkalden, Germany \\ ${ }^{2}$ Circadian Technologies, Inc., Stoneham, Massachusetts, USA \\ ${ }^{3}$ Institute for System Analysis and Applied Numerics, Tabarz, Germany \\ ${ }^{4}$ Work and Organizational Psychology, University of Wuppertal, Germany \\ Email: m.golz@fh-sm.de
}

\begin{abstract}
Summary: Periodogram and other spectral power estimation methods are established in quantitative EEG analysis. Their outcome in case of drowsy subjects fulfilling a sustained attention task is difficult to interpret. Two novel kind of EEG analysis based on pattern recognition were proposed recently, namely the microsleep (MS) and the alpha burst (AB) pattern recognition. We compare both methods by applying them to the same experimental data and relating their output variables to two independent variables of driver drowsiness. The latter was an objective lane tracking performance variable and the first was a subjective variable of self-experienced sleepiness. Results offer remarkable differences between both EEG analysis methodologies. The expected increase with time since sleep as well as with time on task, which also exhibited in both independent variables, was not identified after applying AB recognition. The EEG immediately before fatigue related crashes contained both patterns. MS patterns were remarkably more frequent before crashes; almost every crash (98.5 \%) was preceded by MS patterns, whereas less than $64 \%$ of all crashes had AB patterns within a 10 sec pre-crash interval.
\end{abstract}

\section{INTRODUCTION}

A novel kind of EEG analysis for drowsiness detection has been proposed by several authors during the last decade. The assumption of long-term stationarity of the EEG has been dropped and in lieu thereof patterns on the time scale of several seconds has been analyzed.

The assumption of stationarity of a random process requires temporally constant statistical properties of the EEG. In the weak sense of stationarity the first and second moment, i.e. mean and variance, of the amplitude probability distribution have to be constant. This is by far not fulfilled in several cases when subjects have to fulfill tasks, like steering a car and tracking the lane, whereas fighting against sleep pressure. In such cases the EEG reflects a lot of different ongoing and disappearing processes as well as of fluctuations in vegetative regulation. More than 40 years ago Maulsby et al. (1968) stated in their report of a large normative study involving 200 male subjects that the EEG of drowsiness was found to have "infinitely more complex and variable patterns than the wakeful EEG pattern”. Also Santamaria \& Chiappa (1987) reported in their review paper on different drowsiness related EEG patterns. They inspected visually the EEG grapho-elements and stated: "There is a great deal of variability in the EEG of drowsiness among different subjects”. Many reports on quantitative EEG analysis of drowsy subjects did not consider these patterns and the large variability between subjects. Other authors investigated EEG with modern nonlinear signal processing methods or localized time-frequency methods, but didn’t searched for distinct drowsiness related patterns within the whole recordings. 
An alternative way of quantitative EEG analysis is to leave the assumption of stationarity over periods of several ten seconds and to search for quasi-stationary patterns on the scale of several seconds. At the psychophysiological level of consideration this means departing the direct characterization of states of vigilance and turning towards short events. They might be caused by short phases of dysregulation in the autonomic nervous system or transitions to other brain states. The advantage of event-based EEG analysis lies in the above mentioned lower restrictions to signal stationarity. In addition, inter-individual differences in the signal characteristics are not such important when pattern characteristics are not too strictly defined. Another advantage is the high temporal resolution in the EEG analysis. Therefore, precise temporal relations to other events, like e.g. behavioral events or crashes, are now allowed to be investigated. Disadvantages coming with this kind of analysis might be the loss of all-encompassing EEG characterization. This contribution aims at comparing two different methods of pattern recognition in the EEG of drivers in order to detect drowsiness. Both methods are compared in terms of their correlation to two independent variables, i.e. the subjective self-ratings of sleepiness on the Karolinska Sleepiness Scale and the objective performance variable SdLat, the standard deviation of lateral position in lane. Moreover, the relation of both patterns to crashes is investigated. It is expected that both drowsiness related EEG patterns occur more frequently immediately before crashes. Firstly, experiments will be described in the next section. Both methods of pattern recognition are shortly described afterwards.

\section{EXPERIMENTS}

In order to compare both pattern recognition methodologies data were drawn from an experimental study conducted between February and May 2007 in our driving simulation lab. The study was designed to investigate driving performance and subjects' behaviour under high level of monotony and sleepiness. Subjects were instructed to track the lane as best as possible and to avoid falling asleep. After returning from MS events subjects were reminded that if driving performance becomes too bad or signs of behavioral MS returned too fast, the experiment would be terminated. When crashes appeared an extensive soundscape as well as video scene was played to increase the emotional importance of this event, to set a short break in the run of the driving simulation and to caution the driver.

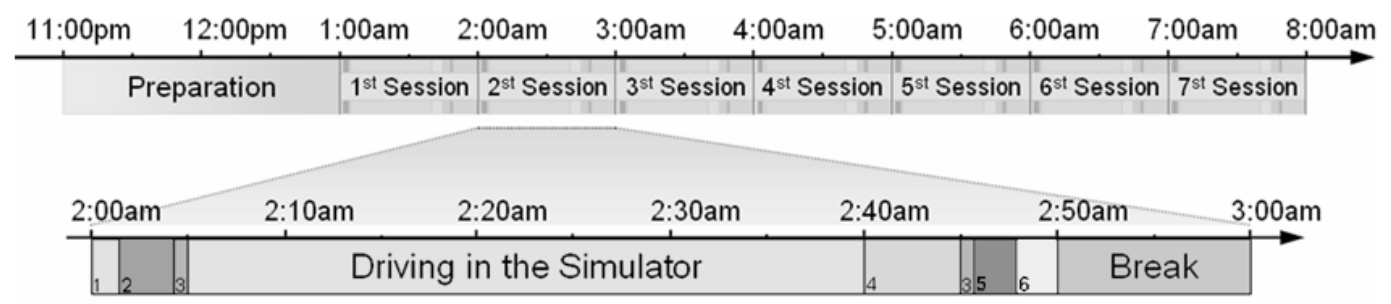

Figure 1. Schedule of one overnight experiment.

Driving simulations started hourly with preceding and subsequent vigilance tasks.

Time since sleep ranged between 16 and 22 hours (from beginning of the $1^{\text {st }}$ to the end of the $7^{\text {th }}$ session)

\section{Subjects}

Participants were 10 students of our university ( 2 females, 8 males) randomly selected out of a set of volunteers who registered their interest. All owned their driving licence for at least 1 year. 
Their age ranged between 19 and 32 years (mean 22.4, SD 4.1). Subjects provided written informed consent and were compensated. They had to perform overnight driving simulations (7 x 40 min) after at least 14 hours time since sleep (Fig. 1). Before and after driving session several questionnaires and vigilance tasks were requested which are reported elsewhere. Breaks between each driving sessions proved to be necessary to motivate the subjects to proceed with the next driving session and to resist increasing sleep pressure.

\section{Measurements}

During the whole night EEG (F1, F2, C3, Cz, C4, O1, O2, A1, A2, com.av.ref.), EOG (vertical, horizontal), ECG, and EMG (m. submentalis) and video recordings of driver's head \& pose, driver's eyes, and the driving scene were recorded. Input variables of the driving simulator, e.g. steering angle, pedal movements, as well as output variables, e.g. velocity, lateral position in lane, were also recorded. During the driving task subjects rated their experienced sleepiness every 4 min on the Karolinska Sleepiness Scale (KSS) (Åkerstedt, 1990). Further experimental details have been published elsewhere (Golz et al., 2007).

\section{METHODS}

Both pattern recognition methods are distinguished by high temporal resolution, approaches for signal-noise-separation, and robustness. Their outcome is the ratio between accumulated pattern duration and length of the accumulation interval (e.g. $2 \mathrm{~min}$ ). The ratio of the accumulated length of alpha burst (AB) and microsleep (MS) patterns to the length of the accumulation interval is denoted here as the $\mathrm{AB}$ percentage (ABP) and the MS percentage (MSP), respectively.

\section{Automatic detection of alpha bursts (AB) patterns in the EEG}

The methodology for AB detection utilized in our investigations was proposed by Schmidt et al (2011). It requires sufficient signal to noise ratio of the recorded EEG. Authors stated that analysis of one single EEG channel is sufficient to detect AB, but even so they applied their method to a lot of EEG channels in parallel. AB should be discrete events which should be remarkably visible in the EEG. They were defined by a) duration (at least $0.5 \mathrm{~s}$ up to several seconds), b) spectral power density (narrowband characteristics), and c) stable peak frequency (in the alpha range, maximal shift $<10 \%)$. AB were not defined by any kind of an amplitude criteria. The advantage of this kind of definition lies in the lower sensibility to noise. It is suggested to remove artifacts before the following steps are performed.

The following steps were performed. EEG was decomposed in overlapping segments (1s length, $75 \%$ overlap). After that the standard direct spectral estimation (trend elimination, Hamming window, Welch method) was applied and the resulting power spectral densities (PSD) were analyzed if their maximum lay within the frequency range of 7 and $13 \mathrm{~Hz}$ (extended alpha range). Then the full width at half maximum (FWHM) was calculated and it was checked if it was lower than two times the bandwidth of the Hamming window applied for spectral estimation. An exponential model is fitted to the actual and to the mean PSD spectrum. This way, the signal PSD as the area above and the noise PSD as the area below the fitted curve can be estimated. This is needed for amplitude normalization to compensate varying noise levels. Only EEG segments 
having signal energy within the FWHM interval at least twice as large as the noise energy and having a relative peak frequency shift lower than $10 \%$ were recognized as AB pattern.

\section{Automatic detection of microsleep (MS) patterns in the EEG}

The automatic recognition of MS patterns utilizes machine learning algorithms, namely supportvector machines, and incorporates human judgements of signs of extreme sleepiness. A rough outline is presented below; details were published elsewhere (Golz et al. 2007).

MS judgement. Two operators who watched the video streams performed a first judgment of ongoing MS by observating the driving simulations and inspecting subject's behaviour while watching the video streams. Typical signs of MS are prolonged eyelid closures, slow roving eye movements, head noddings, slow drifting head movements, and major driving incidents. They are often followed by abrupt reactions. All recorded video material and biosignals underwent offline scoring made by an independent and trained rater. He refined and eventually corrected the online ratings. This resulted in relative precise points in time where MS started; in average 0.8 MS events per minute, in total 2,290 MS events were observed (Golz et al, 2009). MS detection. EEG and EOG signals surrounding these behavioral MS were segmented beginning $3 \mathrm{~s}$ before and ending $3 \mathrm{~s}$ after the MS starting point. PSD was estimated ( $1^{\text {st }}$ order detrending, Hann window, modified periodogram method) and logarithmically scaled. LogPSD was summed in $1 \mathrm{~Hz}$ wide spectral bands in the range from 0.5 to $23 \mathrm{~Hz}$. Further signal features were extracted utilizing a state space method, namely the delay-vector variance (DVV), (Gautama, Mandic, Van Hulle, 2004). Support-Vector Machines (SVM) with Gaussian kernel function (Vapnik 1998) were applied to the summed logPSD and DVV data. During machine learning SVM was trained to separate MS from Non-MS, solely based on the processed EEG and EOG segments. The training involves optimization of several internal parameters and consumes ca. $10^{6}$ times more computational time than simple pattern recognition methods. This resulted in an accurate MS detector with mean accuracy of $97.7 \%$ on evident examples of behavioral MS. MS detector recall. The MS detector has been applied consecutively (6 s segment length, $0.1 \mathrm{~s}$ step size) to the whole EEG/EOG recordings in order to detect further MS-like patterns in the EEG/EOG where in the video streams no clear behavioral events were observable. This resulted in an increase of the number of MS patterns; a total of 3,250 MS patterns without behavioral signs of MS and a total of 2,290 MS patterns in conjunction with behavioral events were found.

Table 1. Similarities and dissimilarities of both pattern recognition methods

\begin{tabular}{|c|c|c|}
\hline Method & AB detection & MS detection \\
\hline Processed Signals & EEG, 1 channel (O1) & EEG (F1/2, C3/4, Cz, O1/2, A1/2), EOG \\
\hline Temporal resolution & High (0.25 s) & High (0.1 s) \\
\hline Detected pattern duration & Ca. $0.5-3 \mathrm{~s}$ & Сa. $1-12 \mathrm{~s}$ \\
\hline Pattern characterization in & Spectral domain & Spectral domain \& State space \\
\hline Pattern definition type & Fixed & Adaptive \\
\hline Pattern recognition & $\begin{array}{l}\text { Fixed decision rules utilizing } \\
\text { threshold values }\end{array}$ & $\begin{array}{l}\text { Machine learning, } \\
\text { Support-Vector Machines }\end{array}$ \\
\hline Artifact removal & Comprehensive & Comprehensive \\
\hline Signal - noise separation & Yes, exponential fit & None \\
\hline Output & \multicolumn{2}{|c|}{$\begin{array}{l}\text { Pattern probability, i.e. ratio between accumulated pattern duration and length of the accu- } \\
\text { mulation interval }\end{array}$} \\
\hline
\end{tabular}

\section{Comparison between both pattern recognition method}

Table 1 provides a summary of both methods. The main difference lies in the definition of the patterns. AB were predefined with fixed rules whereas MS were not defined. The human rater 
depicts points in time where behavioral signs of MS exhibited and afterwards the algorithms had to learn the characteristics of the associated EEG/EOG patterns. Another main difference comes with the classification rule. AB were recognized by threshold rules which restricts the separation function to be linear and parallel to axes in feature space. MS were detected utilizing a nonlinear separation function with many degrees of freedom.
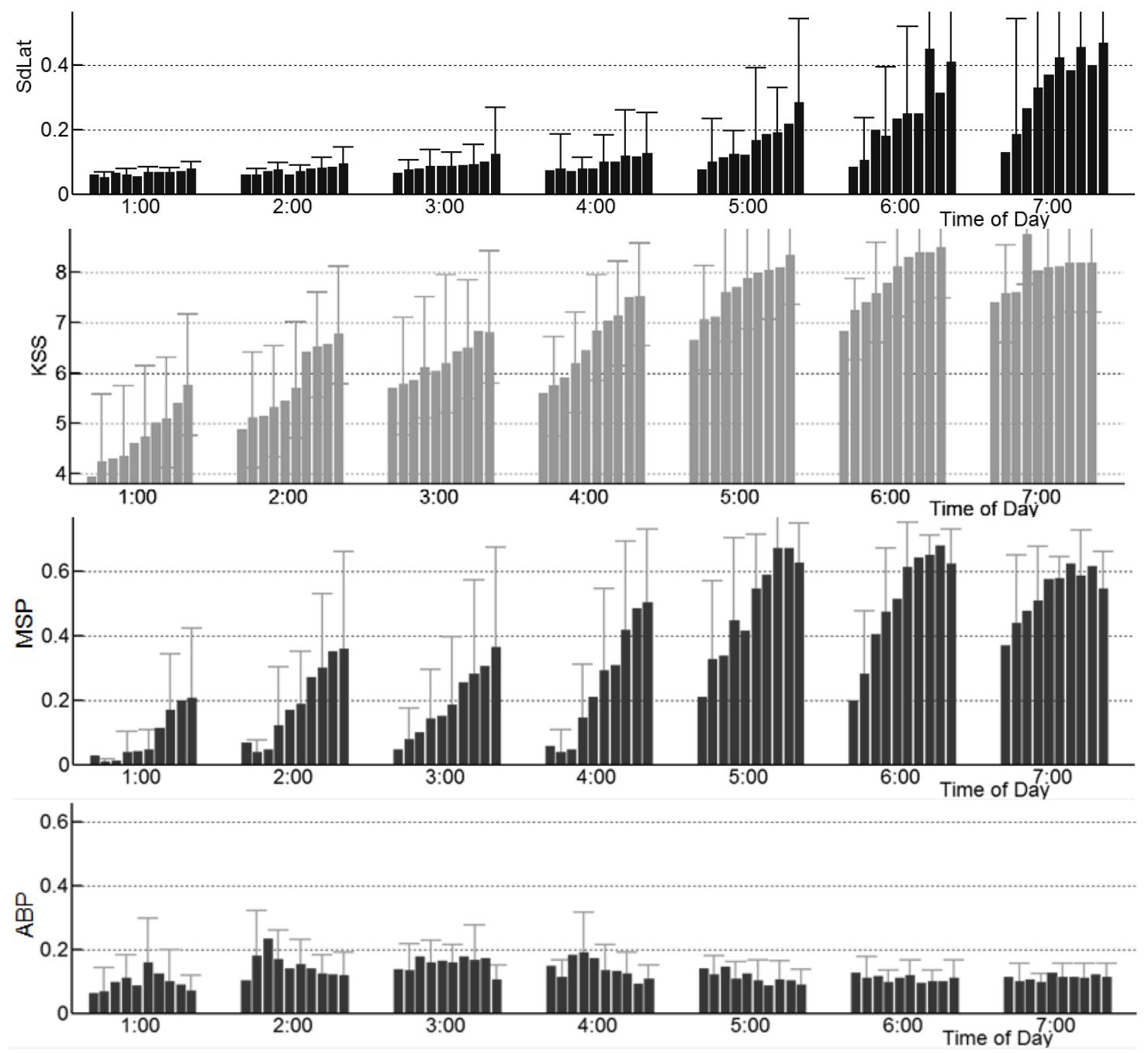

Figure 2. Results of 7 overnight driving sessions, averaged across subjects. Mean and standard deviations of two independent drowsiness variables (SdLat, KSS) as well as two detector output variables (MSP, ABP). All variables, but not $\mathrm{ABP}$, display strong time on task as well as strong time since sleep effects

\section{RESULTS}

For each driving session (40 min) the independent variables as well as both detector output variables werde averaged within 4 min intervals. Afterwards, the mean and standard deviations were calculated across all subjects (Fig. 2).

Driving performance. The independent, objective variable SdLat (standard deviation of the lateral position in lane) turned out to be relatively low during the first 4 sessions and having a time on task effect, i.e. an increase of SdLat with increasing driving time. During the last 3 sessions SdLat increased rapidly, both in mean and standard deviation. 
Self-rating on the Karolinska Sleepiness Scale. The independent, subjective variable KSS increased within and between every session, with one exception. Between the second half of the $6^{\text {th }}$ and the $7^{\text {th }}$ session a slight decrease appeared. It is not clear whether circadian rhythmicity or motivational effects (approaching end of duty) or both were the underlying causes.

Output of microsleep detection. The resulting MS percentage (MSP) displayed the same strong effects with time on task as well as with time since sleep as both independent variables. As early as in the $1^{\text {st }}$ session MS patterns were already detected. This was somewhat unexpected because very low numbers of behavioral signs of MS were detected simultaneously. At the end of the night MSP decreases slightly similar to KSS.

Output of alpha burst detection. The percentage of AB patterns (ABP) increased from the $1^{\text {st }}$ to the $2^{\text {nd }}$ driving session and decreased slightly during the second half of the night. No time on task as well as no time since sleep effect could be observed. ABP had a much lower variability between subjects (low standard deviations) than the other three variables. The mean number of $\mathrm{AB}$ events ranged between 188 and 309 and was more than twice higher than the mean number of MS events (range: $41-145$ ). Crashes were seldom within the first 4 sessions. They increased remarkably between the $5^{\text {th }}$ and $7^{\text {th }}$ session (range: $0-45$ ).

The length of AB events remained nearly constant with time since sleep (ca. $1.2 \mathrm{~s}$ ). The length of MS events increased monotonously from the $1^{\text {st }}$ to the $5^{\text {th }}$ session (range: $4.9 \mathrm{~s}-9.3 \mathrm{~s}$ ) and decreased slightly within the $6^{\text {th }}$ and $7^{\text {th }}$ session.

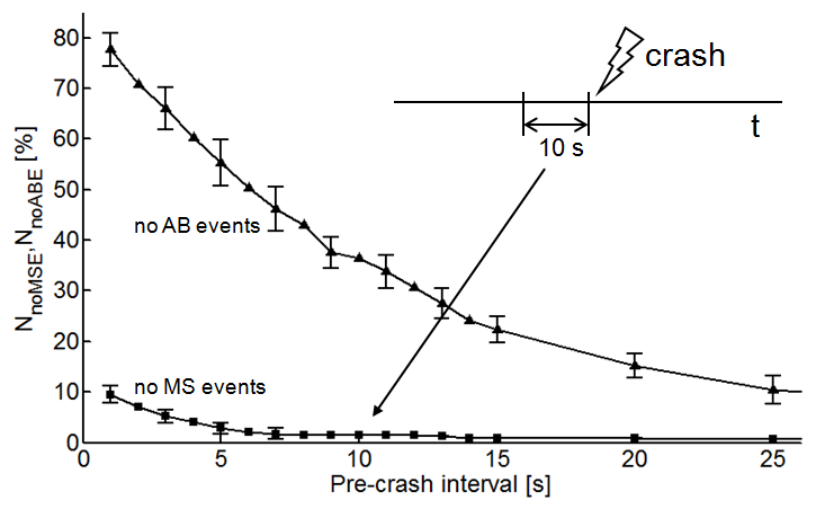

Figure 3. The percentage of pre-crash intervals containing no MS events is much lower than for AB events

Results of crash related analysis. Driving performance degradations leading to events with four wheels out of lane were regarded as crashes. They were rare events; within the first four sessions almost no crash was observed. From the $5^{\text {th }}$ to the $7^{\text {th }}$ session they became likelier; their occurrence (within the 40 min driving sessions) averaged across subjects was 8.8, 28.3, and 44.9 respectively. Before every crash it was investigated how often the worst case of no remarkable EEG pattern eventuated. We assumed a pre-crash interval of $10 \mathrm{~s}$; enough time would remain to initiate effective countermeasures. It turned out that nearly no crash with missing MS patterns occurred; only $1.49 \%$ of all crashes were not accompanied by MS pattern in the EEG/EOG (Fig. 3). In contrast, in $36.5 \%$ of all crashes no AB patterns were found within the 10 s interval immediately before the crash. 


\section{DISCUSSION}

This comparative investigation of two EEG pattern recognition methodologies found remarkable differences. AB pattern appeared relatively often and with low duration. They had no significant time on task as well as no time since sleep effect. This is in contrast to the temporal development of behavioral signs of drowsiness as well as with both independent variables of drowsiness. The increase of $\mathrm{AB}$ percentage within the first half of the night might be interpreted as early indications of sleepiness. During the second half of the night where driver's performance dramatically deteriorated AB remained insensitive. Moreover, the results of crash analysis showed that AB seemed to arise spontaneously and not strictly related to degradations due to drowsiness. This is in contrast to Schmidt et al (2011) who observed an increase of the AB rate from $7 \mathrm{~min}^{-1}$ to 12.8 $\min ^{-1}$ when the $1^{\text {st }}$ and the last 20 min of their driving sessions were compared. They reported also an increase of AB duration from $0.62 \mathrm{~s}$ to $0.665 \mathrm{~s}$ which we couldn't verify. AB patterns turned out to be a deal worse as forerunners of crashes. In more than a third of all crashes no $A B$ were found immediately before.

\section{CONCLUSIONS}

This study indicates that MS patterns in the EEG/EOG detected with machine learning algorithms have remarkable correlations with self-rated sleepiness as well as lane tracking performance. They are reliable forerunners of fatigue related crashes. The percentage of AB patterns increased in the early phases of drowsiness development. Their connection to crashes seems to be loose.

\section{REFERENCES}

Åkerstedt, T. (1990). Subjective and Objective Sleepiness in the Active Individual. Int J Neurosci, 52, 29-37.

Gautama, T., Mandic, D.P., \& Van Hulle, M.M. (2004). Novel Method for Determining the Nature of Time Series, IEEE Transactions on Biomedical Engineering, 51, 728-736.

Golz, M., Sommer, D., Krajewski, J., Trutschel, U., \& Edwards, D. (2009). Microsleep Episodes and Related Crashes during Overnight Driving Simulations. Proc. $6^{\text {th }}$ Int Sympos Driver Assessment, Olympic Valley - Lake Tahoe, California, 39-45.

Golz, M., Sommer, D., Chen, M., Trutschel, U., \& Mandic, D. (2007). Feature Fusion for the Detection of Microsleep Events, J VLSI Signal Proc Syst, 49, 329-342.

Maulsby, R.L. et al (1968). The Normative Electroencephalographic Data Reference Library, Final Rep., Contract NASA 9-1200, National Aeronautics \& Space Admin., Washington D.C.

Santamaria, J., \& Chiappa, K.H. (1987). The EEG of Drowsiness in Normal Adults, J Clin Neurophysiol, 4, 327-382.

Simon, M., Schmidt, E.A., Kincses, W.E., et al. (2011). EEG Alpha Spindle Measures as Indicators of Driver Fatigue under real Traffic Conditions, J Clin Neurophysiol, 122, 1168-1178.

Vapnik, V.N. (1998). Statistical Learning Theory. New York: Wiley. 